\title{
Breast Cancer Research in Iran: A Scientometric Analysis of Publications Output from 1991 to 2015 in Scopus
}

\author{
Alireza Gharib ${ }^{1, *}$ \\ ${ }^{1}$ Student Research Committee, Hamadan University of Medical Sciences, Hamadan, \\ Iran \\ * Corresponding author: Alireza Gharib, Student Research Committee, Hamadan \\ University of Medical Sciences, Hamadan, Iran. Tel: +98-9123862306, E-mail: \\ gharibalireza@yahoo.com
}

DOI: $10.21859 /$ mci-supp- 80

\section{Keywords:}

Bibliometrics

Breast Cancer

Bibliographic Database

Scopus

Iran

\begin{abstract}
Introduction: As a common type of malignancy, breast cancer is one of the major causes of death in women globally. The purpose of the current study was to analyze Iran research performance on Breast Cancer in the context of national and international studies, shown in the publications indexed in Scopus database during 1991-2015.

Materials and Methods: Data were retrieved from the Scopus citation database in this scientometric study. The following string was employed; "breast cancer OR breast malignancy OR breast tumor OR mammary ductal carcinoma" keywords in the main title, abstract and keywords and Iran in the affiliation field were the main related keywords. The terms used were searched in Scopus using the tab specified for searching documents. Time span analyzed was 1991 to 2015 inclusive. Using the analyzing software of Scopus, we analyzed the results.

Results: Iran's increasing publication production during 1991-2015 in breast cancer research which indexed in Scopus, consists of 2,399 papers with an average of 95.96 papers per year, and achieved an h-index of 48. Iranian cancer research articles have received 15,574 citations during 1991-2015, and average citations per paper were 6.49. Iran ranked 27 th among the top 30 nations with a worldwide stake of $0.67 \%$, the 20 top publishing journals published 744 (31\%) Iranian research articles on breast cancer, among them, there were 15 Iranian journals.

Conclusions: The number of Iranian research papers on breast cancer and also the number of citations to them, is increasing. Although the quantity and quality of papers are increasing, regarding the prevalence of breast cancer in Iran and also the ineffectiveness of screening programs in the early detection of the cases, more effort should be made, and Iranian policy makers should consider more investment on breast cancer research.
\end{abstract}

